\title{
Binge eating disorder: general practitioners' constructs of an ambiguous pathology
}

Elizabeth Henderson School of Primary Care, University of Manchester, Manchester, UK, Carl May Centre for Health Services Research, University of Newcastle upon Tyne, Newcastle, UK, and Carolyn A. Chew-Graham School of Primary Care, University of Manchester, Manchester, UK

\begin{abstract}
Eating disorders are amongst the most commonly encountered psychiatric disorders experienced by young women. Binge eating disorder (BED) has received some support as a distinct pathology, but is hard to disentangle from other kinds of behaviours. This qualitative study explored awareness and knowledge of BED amongst a group of 18 inner-city general practitioners in NW England. Thematic coding of their accounts suggested a dichotomous tension. (1) Subjects were largely unaware of the existence of BED, and found it difficult to conceptualize its diagnosis and management in primary care. (2) Subjects framed BED as a 'disorder' that was firmly within the sphere of patients' personal responsibility, and recognized that psychological distress would be an important causal factor in its aetiology. Subjects were reluctant to consider BED as a diagnosis for obese patients because of the absence of services for onward referral, and because of uncertainties about effective treatment.
\end{abstract}

Key words: anorexia; binge-eating; bulimia; eating disorders; obesity

\section{Introduction}

Eating disorders are amongst the most commonly encountered psychiatric disorders experienced by young women. The prevalence of this group of disorders, including anorexia and bulimia nervosa appears to have increased in recent years (Van't Hof and Nicolson, 1996). Early detection is important in avoiding associated physical and psychosocial problems. However, sufferers often work to conceal their state and consequent delays in diagnosis and treatment are common, up to $50 \%$ of cases may remain undiagnosed. Existing services and research efforts are directed primarily at disorders, such as anorexia and bulimia (Hsu, 1996).

There are however, a bundle of much more ambiguous eating disorders, of which binge eating disorder (BED) is one. It is claimed that this condition is characterized by obesity, sustained and compulsive overeating, without compensatory

Address for correspondence: Carl May, Centre for Health Services Research, University of Newcastle upon Tyne, 21 Claremont Place, Newcastle upon Tyne, NE2 4AA, UK. Email: c.r.may@ncl.ac.uk purging (Mizes and Sloan, 1998; Mizes, 1998). $\mathrm{BED}$ is therefore an eating disorder where weight gain rather than wasting is the principal clinical sign. BED was assigned a provisional set of diagnostic criteria in DSM-IV, but its prevalence is uncertain, and there is a similarly limited literature on the phenomenology and management of this disorder (de Zwaan, 2001; Devlin, 1996; Spitier et al., 1991).

Psychosocial aspects of aetiology, management and recovery in eating disorders have attracted much attention. Much less attention has been paid to the everyday views of clinicians, however, even though they are crucial in forming the point of departure for effective diagnosis, treatment and management. General practitioners are crucial to the effective diagnosis and treatment of BED, since they form the primary pathway into medical care for sufferers. However, we know nothing about the ways that general practitioners conceptualize the boundary between 'normal' and 'pathological' obesity. The qualitative analysis reported here was designed to explore (a) general practitioners' knowledge of BED, its diagnosis and management, and (b) the ways that knowledge about BED related to wider problems around obesity. While 
there is a clinical push to promote BED as a diagnostic category, this reflects endemic obesity in the US (Dingemans et al., 2002). So in the UK the relevance of the pathology to practitioners is in doubt. For us, the diagnostic category had utility at another level. We were interested in how the boundaries of the normal and the pathological are defined in primary care medicine. BED is a useful vehicle.

\section{Study group and method}

Because there is no existing literature on primary care clinicians' attitudes to BED, we undertook an exploratory qualitative study. The study was conducted in June and July 2000. A convenience sample was obtained by contacting all general practitioners with honorary lecturer appointments at a British medical school in NW England by letter and inviting them to take part. Eighteen general practitioners (nine female and nine male) agreed to do so within the sampling time frame. Interviews were conducted by EH. These were informal - lasting around $45 \mathrm{~min}$ - and subjects consented to their being audiotaped. Audiotapes were transcribed verbatim and the transcripts, along with contemporaneous notes of the remaining interview, comprised the data subjected to formal analysis. Data analysis followed the broad principles of constant comparison set out by Strauss (1987): interview transcripts were read, and thematic coding undertaken iteratively. Themes were pursued developmentally during the course of the study, with the interview schedule being modified as emergent categories of data were identified. Initial coding was undertaken by $\mathrm{EH}$, and intepretation and analysis of data was undertaken by all three authors. Category saturation was achieved in two of these: (a) relating to the difficulty of identifying eating disorders in the obese; and (b) relating to the culpability of patients. Our analysis of these two saturated thematic categories is presented in this paper.

\section{Eating disorders in the obese}

The first aim of the study was to understand how general practitioners conceptualize BED. All subjects were asked what, if anything, they understood by the term 'binge eating disorder'. None of them were able to answer this question confidently, and most were unaware of its existence as a distinct diagnostic category. Five subjects were able to ascertain that BED referred to those who binge eat without purging.

GP1: ... in my mind I'd categorize it as binge eating without auto-vomiting.

GP18: That will be people who for one reason or the other, of psychological problems, find solace in eating, and they go and eat whatever they can, wrong eating, just to satisfy whatever their other, their underlying problem is.

Half $(9 / 18)$ of the sample gave a definition of what appeared to be bulimia nervosa, and many admitted that they were unable to differentiate the two.

GP16: Well I presume it means the patients who eat too much and then make themselves sick to part with it and to maintain their normal weight.

GP11: I thought that was similar to bulimia. In fact I haven't made any distinction in my mind, so my understanding was that binge eaters also vomit, make themselves vomit, and that those are the bulimics.

The remaining four respondents did not know what BED was. When subjects were shown the DSM-IV criteria for BED, however, a number felt that they had experience of patients who would fall into this category.

GP6: Oh yes, indeed. We've got a few grossly fat people, well a handful who are you know - pathologically obese and must fall into this category.

One doctor, who had referred a similar patient to the eating disorders service for treatment, was unsure of the purpose of a diagnostic label for such patients. For a subgroup of GPs who are conscious of the potential link between a person's psychological state and compensation through eating behaviours, the introduction of a name or title may be regarded as unnecessary. They may manage the condition appropriately regardless of whether certain distinguishing behaviours are present or not. 
GP8: I don't think I was aware that it was a specific classification on its own. I'm not actually sure that it's very helpful, because to me it's just a shade different from what I'd call bulimia - it would need the same approach really in terms of helping someone whether they vomit or not.

Other subjects also pointed to the difficulty in managing such patients because of the dysfunctional behaviour that led to compulsively overeating and consequent obesity.

GP2: They're very difficult to treat because they don't want to be fat, and yet they continue to binge eat, and behaviour modification's something that's very hard to do.

GP14: Unless you change their lifestyle, you're not really going to treat their depression or relieve their depression. In a few patients that are like that we do treat them with antidepressants, but they tend to gain weight with them. So it's a vicious cycle, it's very difficult, and I don't know really how to address it except plugging away at lifestyle.

All subjects were also asked why they thought that BED appeared to be less widely recognized than the other eating disorders. Some felt that part of that reason was the difficulty in managing the problem, as discussed above. They saw little point in providing a label for a condition that could not be effectively managed.

GP4: I mean there's lots of overweight people as well, so you worry about opening a can of worms if you try to dig into psychological causes.

GP6: I think GPs are pragmatic people, and if there's something that you can't do much about then you're not really going to go out of your way to find it.

In this context, pragmatism extended to the difficulty of disentangling BED from the wider problem of obesity. The likelihood of presentation or detection of this disorder was influenced by the limits that general practitioners imposed on themselves in their attempts to achieve behaviour change.
GP12: [It is] less of a challenge because it's very common, and there's less, I feel there's less I can do in my role in terms of changing their lifestyle, and it's more about society, and well, probably a greater influence of society, of attitudes.

Other subjects felt that BED is less well recognized than anorexia or bulimia because its physical effects are less obvious or striking, again as a result of the growing prevalence of obesity in our society.

GP4: Well I think something like anorexia is, you know, physically it's very obvious.

GP13: Anorexia and bulimia have a quick phenotypic effect which is consistent and has consequences. In binge eating the effects are not noticeable as quickly so not as recognized.

Despite the wide range of physical morbidity that is associated with being overweight, some felt that this disorder is somehow less legitimately a medical problem than anorexia or bulimia.

GP5: Where as bulimia nervosa, it tends to be more medicalized because of the other features which appear more abnormal to relatives.

In this context, subjects suggested that many such patients would be dismissed as cases of 'simple' obesity without further exploration of underlying factors. Clearly this has consequences for rates of diagnosis and its wider recognition.

GP4: I think generally being overweight is something we don't tackle very much and therefore probably just don't delve into it.

GP14: I would say it's not something that we have a lot to do with simply because people don't really, you know, if they're a bit overweight, they don't really think that they've got an illness.

The lack of awareness of BED amongst general practitioners suggested by these accounts may mean that its true prevalence is concealed. But more importantly, the wider difficulty of disentangling BED from other forms of obesity in the primary care consultation needs to be understood. 


\section{Obesity as a problem of culpability}

The second objective of our analysis was to locate BED in its wider social context. Obesity was widely regarded as being an increasing problem in general practice and one that is difficult to manage efficiently. A lack of resources is one limitation in providing obese patients with effective advice, support and follow-up. This leaves the GP feeling powerless and ineffective in what they can offer the patient who is battling to lose weight.

GP18: They have a lot of struggle to lose weight, but they don't succeed. They ask me the question, and I don't have a clear cut, convincing answer for them. In other words I'm not capable of dealing with it.

Beyond the constraints of resources, several respondents felt that the nature of the obesity problem restricted what they could offer to the patient. Attitudes towards food and eating habits are part of the individual's lifestyle and often involve rigid behavioural patterns, influenced by family, socioeconomic class and culture.

GP4: You don't feel you can do an awful lot about it. You can give dietary advice and discuss exercise, but it can be very engrained peoples' eating habits. It's hard to shift.

GP14: I try and encourage as much as possible in obese patients to exercise more and to eat less, but it is very difficult because you're talking of changing their lifestyle, and that, we have been shown time and time again in general practice, it's lifestyle changes that are difficult.

As a result, feelings of frustration, despondency and even irritation were expressed by several interviewees when discussing the management of obesity.

EH: so how do you feel when an obese patient comes and says they want to lose weight?

GP10: That probably invokes more feelings of despondency in me [than eating disorders] because I do feel that, you know, we haven't got a great deal to offer them because there are a lot of time constraints that you work under, and I don't think that my time is best used being a kind of Weight Watchers for a single individual.

A further barrier in the management of obesity appears to be a reluctance to discuss weight, on the part of either the doctor or the patient.

GP1: I suppose, I um, I don't often mention weight unless either the patient raises it or it's strongly linked to something else.

GP15: I think it's again an area that I think the patients are very sensitive about, it's an area that uh, overweight patients are extremely sensitive about and very reluctant to discuss with their family doctor.

This aversion to discussing weight hinders further the possibility for the exploration of underlying causes of overeating. If that underlying cause is psychological, the patient, one would expect, is unlikely to initiate such a discussion. As well as the sensitivity required to approach the issue of weight, discussion may be further hindered by the defensive attitude of the patient.

GP4: But the other thing is that you also get a resistance sometimes if you talk to people about their weight, that they say they hardly eat anything, you know, and where do you go from there really?

Other issues that arose when discussing obesity, and which may influence management choices, involved subjects' ideas about the patients' personal responsibility for health. Stigma surrounding obesity is widely documented, and the belief that weight gain is a problem of personal agency and self-discipline, rather than a 'medical' problem is commonplace. This was reflected in subjects' accounts, which addressed the role of personal responsibility in the development of obesity.

GP4: You do feel that it's something that, it's their fault. They've eaten too much and they're not doing any exercise, and so they are overweight.

GP8: I suppose it's like people who smoke and complain of a cough in a sense. When there's a behaviour attached to something, you feel that people ought, 'ought', to be able to control it. 
The perception that obese patients were to some extent culpable for their condition is reflected in the extent to which subjects were willing to offer support for their patients. It was felt that the patient was responsible for the underlying behaviour that led to obesity, and that they were responsible for initiating behavioural change.

GP17: I think you've got to get the patient to understand that it's their behaviour that's got to change, and it's they who are in control, not you as the doctor.

This issue of control over patterns of health behaviour provoked resentment and feelings of frustration among some subjects. They were willing to offer dietary and exercise advice and highlight the risks of physical morbidity, but were keen to point out that responsibility for the situation ultimately lay with the patient.

GP6: I think that you can get them to acknowledge that it's actually their problem and not your problem. Some people want to, you know, 'there you are doctor, it's your problem, you must stop me eating', but of course at the end of the day, they're the person in charge of the biscuit barrel or whatever.

GP14: You have to at the end of the day say, 'well, this is your problem, I can't do anything about it. Why is it a medical problem that you eat too much?'

Despite these kinds of moral judgement about the patient's trajectory, subjects recognized that underlying psychological problems were important causal factors in obesity and were frequently able to give examples of such patients.

GP5: Yeah, I think it's quite common. I mean, a lot of people are comfort eaters. They might be mildly anxious or, well most of the time mildly anxious or mildly depressed, but if they come to a crisis with which they can't cope then they go off and binge, you know.

Similar observations regarding depression in the obese were commonly made during interviews, and some subjects asserted that it was difficult to distinguish which was the cause and which the effect.
GP7: You address perhaps the comfort eating side of things with them, and very often someone who's got a weight problem will tend to be sort of depressed about that anyway, so it's difficult to know what's causing what. But I'm sure there's a cycle of it, yeah.

GP14: I think a lot of my obese patients are depressed, a lot of them. It's a vicious cycle, they're overweight, they're depressed, so they eat more, very very obviously so, I would say.

One subject elaborated further on predisposing factors and on the potential for secondary gains derived from obesity.

GP8: I think that often it is a sort of defence. I mean if you make yourself fat and unattractive it sort of protects you in a way from a lot of the things that you .... and it becomes, 'oh well, I can't do this because I'm fat and unattractive'. Actually part of that is not actually wanting to or being frightened of it.

The majority of subjects emphasized psychological causes for excessive or compulsive eating in obesity. At the same time, however, they asserted the personal responsibility of the sufferer in resolving the condition.

\section{Conclusion}

Although it is the only one of its kind, this paper reports a small-scale qualitative study that is not fully generalizable to other settings. Nevertheless, subjects in this study are broadly typical of urban general practitioners. The two thematic categories in their accounts that we have discussed are dichotomous:

(a) Subjects were largely unaware of the existence of BED, and found it difficult to conceptualize its diagnosis and management in primary care.

(b) Subjects framed BED as a 'disorder' that was firmly within the sphere of patients' personal responsibility, and recognized that psychological distress would be an important causal factor in its aetiology.

We have observed elsewhere (Chew-Graham et al., 2001; May etal., 1996; Wileman etal., 
2002) that general practitioners describe themselves as increasingly presented with diffuse psychological problems where the boundaries between 'medical' and the 'social' categories are unclear to them, and where existing forms of clinical management are ineffective. The underlying problem that they face, however, is almost certainly not the difficulty that exists in demarcating the boundaries of apparently ambiguous pathologies. The history of medicine in recent years is replete with these shifts being quickly and unproblematically achieved, notably in the case of depression (Shorter, 1992; Stone, 1998).

Far more important in understanding general practitioners' reluctance to accede to this new clinical category is their sense of powerlessness in the face of it. This parallels other areas of clinical practice where pathological entities are well defined, but their resolution seems to depend on the willingness of the patient to accept and act upon a socially uncongenial diagnosis. Such problems would include, for example, somatisation (Salmon et al., 1999), depression, chronic low back pain (May et al., 1999) and alcohol dependence (Thom and Tellez, 1986). Subjects in this study were quite blunt: without an effective means of managing BED, they were unlikely to actively seek opportunities to diagnose it.

\section{Acknowledgements}

We thank the general practitioners who took part in this study for their time and candour. We thank Lindsey Wileman, Scott Hedley and Hannah Cole for their help in the conduct of this project, and gratefully acknowledge secretarial support from Mrs Caroline Connolly and Mrs Denise Mukadam.

\section{References}

Chew-Graham, C.A., May, C., Cole, H. and Hedley, S. 2001: The burden of depression in primary care: a qualitative investi- gation of general practitioners' constructs of depressed people in the inner city. Primary Care Psychiatry 6, 137-41.

Devlin, M.J. 1996: Assessment and treatment of binge-eating disorder. Psychiatric Clinics of North America 19, 761-67.

De Zwaan, M. 2001: Binge eating disorder and obesity. International Journal of Obesity 25, S51-S5.

Dingemans, A.E., Bruna, M.J. and van Furth, E.F. 2002: Binge eating disorder: a review. International Journal of Obesity 26, 299-307.

Hsu, L.K.G. 1996: Epidemiology of the eating disorders. Psychiatric Clinics of North America 19, 681-87.

May, C., Dowrick, C. and Richardson, M. 1996: The confidential patient: the social construction of therapeutic relationships in general practice. Social Rev 44, 187-203.

May, C., Doyle, H. and Chew-Graham, C.A. 1999: Medical knowledge and the intractable patient: the case of chronic low back pain. Social Science and Medicine 48, 523-34.

Mizes, J.S. 1998: Neglected topics in eating disorders: guidelines for clinicians and researchers. Clinical Psychology Review 18, 387-90.

Mizes, J.S. and Sloan, D.M. 1998: An empirical analysis of eating disorder, not otherwise specified: preliminary support for a distinct subgroup. International Journal of Eating Disorders 23, 233-42.

Salmon, P., Peters, S. and Stanley, I. 1999: Patients' perceptions of medical explanations for somatisation disorders: qualitative analysis. British Medical Journal 318, 372-76.

Shorter, E. 1992: From paralysis to fatigue: a history of psychosomatic illness in the modern era. New York: Free Press.

Spitzer, R.L., Devlin, M.J., Walsh, B.T., Hasin, D., Wing, R., Marcus, M.D. et al. 1991: Binge eating disorder - to be or not to be in DSM-IV. International Journal of Eating Disorders 10, 627-29.

Stone, S. 1998: Healing the mind: a history of psychiatry from antiquity to the present. London: Pimlico.

Strauss, A. 1987: Qualitative analysis for social scientists. Cambridge: Cambridge University Press.

Thom B. and Tellez, C. 1986: A difficult business - detecting and managing alcohol-problems in general-practice. British Journal of Addiction 81, 405-18.

Van't Hof, S. and Nicolson, M. 1996: The rise and fall of a fact: The increase in anorexia nervosa. Sociology of Health and Illness $18,581-608$.

Wileman, L., May, C. and Chew-Graham, C. 2002: Medically unexplained symptoms and the problem of power in the primary care consultation: a qualitative study. Fam Pract 19, $178-82$. 